\title{
The modeling of the dust deposits on the surfaces of cyclone scrubber
}

\author{
Michail Vasilevsky ${ }^{1}$, Aleksandr Razva ${ }^{1, *}$, Dmitri Zaharov ${ }^{1}$, and Danil Shabirov ${ }^{1}$ \\ ${ }^{1}$ National Research Tomsk Polytechnic University, 634050 Tomsk, Russia
}

\begin{abstract}
The phenomena at interaction of agglomerate of disperse material with the surface of the inertial device are considered. It is revealed that tension in the interactions between the agglomerate surface can reach values comparable to atmospheric pressure, which is the reason for the formation of solid deposits, what is the reason of formation of strong deposits which, in turn, are defined by the autohesion communications of particles forming agglomerate.
\end{abstract}

\section{Introduction}

Dust removals of gases is an integral part of technological processes in the production of power, chemical, metallurgy, construction, food and other products. One of the most common and reliable methods of cleaning industrial gases from suspended particles is mechanically treated in a dry apparatus. A counter flow cyclone dust collector is a key element in systems of gas purification.

In this apparatus, a vortex is organized in such a way that the disperse phase of the aerosol state at the receiver is converted to a dispersed body in which the particles touch each other and interact with each other. The process of dust separation in the cyclone consists of several stages: swirling flow, concentration and withdrawal of particles from the separation zone, forming a layer in the receiver, removing the collected dust from the receiver. Violation of any of stages leads to poor performance of the collector. One of the reasons for reducing the effectiveness of these devices is the formation of deposits of particles on the enclosing surfaces of the separation space and hanging dust volume dust collector. Entering the gas cleaning system particulate matter consists of aggregates of primary particles, aggregated particles, and is along with the carrier medium of the aerosol. In another state it is in the form of deposits or is a bulk body. Agglomerates are formed as a result of turbulent coagulation, when disrupting the flow of deposits from surfaces [1], when handling materials, with segregation of particles as a result of its transport to different devices [2, 3]. In the cyclone itself, migratory-turbulent and gradient near-wall agglomerations are significant.

\section{Theoretical bases and design values}

\footnotetext{
*Corresponding author: razva@mail.ru
} 
We will consider the phenomena at interaction of agglomerate with a surface. At elastic collision of solid bodies no changes of macroscopic mechanical properties of the contacting materials. In this case, depending on the arising interaction force, the contact area is determined by the Hertz formula, which relates the Poisson coefficients and the elasticity moduli of interacting bodies [4]. In most studies on the impact of elastic bodies assume the lawful hypothesis of the static Hertz problem and accept further that the ratio between the contact force and the convergence remains at impact the same as under static loading. To determine the time duration of impact, solve the problem of the motion of the particle during its interaction with the barrier, and is the same for the duration of the process of deepening particles and rebound. Such interaction is characteristic of integral, rigid particles and the ricochet of particles and abrasive wear of surfaces is his consequence.

The agglomerates are composed of many particles, so are heavier compared to the mass of the particles. Depending on the ratios of the radial and tangential velocity of the agglomerate approaching it to the surface is at different angles (Fig.1). The angle of incidence determines the nature of its interaction with the surface. The sinter has a density several times lower than the density of the particles of which it consists. Therefore ricochet hardly possible. Depending on the angle of interaction and between the agglomerate will either be eroded, disperse and again become stronger, or Vice versa, will condense and form deposits. If sediments are solid, they are not destroyed by the flood. In Fig. 1 shows the trajectory of the agglomerate in the channel and conversion scheme of the agglomerate A (dotted line) in the compacted bundle B.
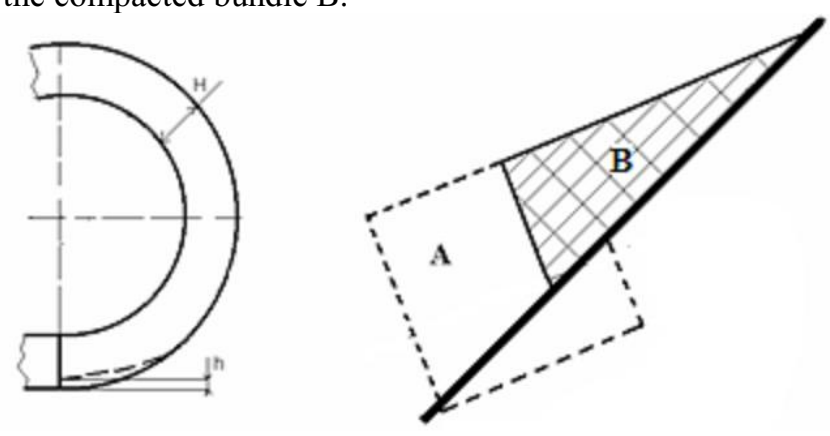

Fig. 1. The trajectory of the agglomerate in the channel and conversion scheme of the agglomerate A (dotted line) in the compacted bundle B.

Agglomerate consisting of small particles, under the influence of centrifugal force moves relative to the periphery of the gas and the interaction with the wall passes through the stages of compaction, deformation, aftereffect. The change of momentum equals the impulse of force generated in the formed contact surface. The interaction time and deformation is determined by the speed of approach of the agglomerate to the wall and the size of the area of "flattening". In addition, can be used the equation of rate of change of energy that binds the kinetic energy and the rate of change of energy of the peripheral forces applied to the deformable surface. All these data allow predicting a state and settings of the agglomerate, if you know the parameters of the layer of particles in the deposition.

The velocity of the particle relative to the gas in curved flow in the radial direction can be found from the dimensionless equations [5].

$$
\frac{\overline{W_{\phi}^{2}}}{\bar{r}}=\Psi \operatorname{Re}_{\delta} \frac{\operatorname{Re}_{\delta}^{2}}{24 S t k \operatorname{Re}_{\delta, \mathrm{w}}},
$$




$$
\begin{aligned}
& \operatorname{Re}_{\delta, \mathrm{w}}=\frac{W_{0} \delta}{v}, \\
& S t k=\frac{\delta^{2}}{18 v} \frac{\rho_{\delta}}{\rho} \frac{W_{0}}{R_{\mathrm{c}}}, \\
& \operatorname{StkRe}_{\delta, \mathrm{w}}=\frac{\rho_{\delta}}{\rho} \frac{W_{0}^{2}}{R_{\mathrm{c}}} \frac{\delta^{3}}{18 v^{2}}, \\
& \operatorname{Re}_{\delta}=\frac{\Delta U \delta}{v},
\end{aligned}
$$

where $\rho, \rho_{\delta}-$ the density of gas and particles, $\delta$ - particle diameter, $v-$ kinematic viscosity of gas, $\Delta U-$ particle velocity relative to the gas in the radial direction, $W_{0}-$ gas inlet velocity, $\bar{W}_{\phi}=\frac{W_{\phi}}{W_{0}}-$ dimensionless peripheral velocity of a particle, $R_{\mathrm{c}}-$ the base value of the radius of the cyclone. In [5] it was shown that in the range $R e_{\delta}=1-100$ the equation (1) can be approximated dependence

$$
\operatorname{Re}_{\delta}=\left(\operatorname{StkRe}_{\delta, \mathrm{w}} \frac{\bar{W}^{2}}{\bar{R}}\right)^{0.75}
$$

(1), but in the range $R e_{\delta}=500-100000$ equation (1) we approximate dependence

$$
\operatorname{Re}_{\delta}=1.7\left(\operatorname{Re}_{\delta, \mathrm{w}}^{2} \frac{\rho_{\delta}}{\rho} \frac{\delta}{R}\right)^{0.5} \text {. }
$$

Let $W_{0}=20(\mathrm{~m} / \mathrm{s}), \frac{\rho_{\delta}}{\rho} \frac{1}{18}=27, R_{\mathrm{c}}=\frac{R_{\text {out }}+R_{\text {inner }}}{2}=\frac{0.4+0.32}{2}=0.36(\mathrm{~m})$, $v=1.5 \times 10^{-5}\left(\mathrm{~m}^{2} / \mathrm{s}\right)$,

$$
\begin{aligned}
S t k & =\frac{\rho_{\delta}}{\rho} \frac{1}{18} \frac{\delta^{2}}{v} \frac{W_{0}}{R_{\mathrm{c}}}=10^{-4} \delta^{2}, \\
\operatorname{Re}_{\delta, \mathrm{w}} & =\frac{w_{0}^{\delta}}{v}=1.33 \delta, \\
\operatorname{Re}_{\delta, \mathrm{w}} & =\operatorname{StkRe}_{\delta, \omega}{ }^{0.75}=12.38 \times 10^{4} \delta^{2.25},
\end{aligned}
$$

where $\delta$ - particle diameter in microns. $\delta_{*}=\left(10^{4} / 1.33\right)^{1 / 3}$. Value $\delta *$ corresponds to the value $R e_{\delta}=1(\mathrm{~m} / \mathrm{s})$.

$$
\Delta U=\operatorname{Re}_{\delta} \frac{v}{\delta}=S t k^{0.75} \operatorname{Re}_{\delta, \mathrm{w}}^{0.75} \frac{W_{0}}{\operatorname{Re}_{\delta, \mathrm{w}}}=W_{0} \frac{S t k^{0.75}}{\operatorname{Re}_{\delta, \mathrm{w}}^{0.25}}=186 \times 10^{-4} \delta^{1.25},
$$


where $\delta$ - diameter of agglomerate particle substitute in microns. And on (7):

$$
\Delta U=\operatorname{Re}_{\delta} \frac{\nu}{\delta}=1.7\left(\operatorname{Re}_{\delta, \mathrm{w}}^{2} \frac{\rho_{\delta}}{\rho} \frac{\delta}{R}\right)^{0.5}=1.7 W_{0}\left(\frac{\rho_{\delta}}{\rho} \frac{\delta}{R_{\mathrm{c}}}\right)^{0.5}=1.249 \sqrt{\delta} .
$$

Agglomerations near the wall pass through a section with a decreasing flow velocity, and at the wall itself the flow velocity is zero. The circumferential velocity of the gas at a distance from the wall $W(y)=\sqrt{\frac{\tau_{\mathrm{w}}}{\rho}}(9.5+1.32 \ln \eta), \eta=\frac{y}{v} \sqrt{\frac{\tau_{\mathrm{w}}}{p}}[6], \tau_{\mathrm{w}}=0.5 C_{\mathrm{f}} \rho W_{\Delta}^{2} / 2$, $C_{\mathrm{f}} \approx 0.006, W_{\Delta}-$ the value of the circumferential velocity at the boundary of the wall zone, $W_{\Delta}=0.95 W_{0}$. Let us assume that on the half of the wall zone of intensive fall of speed $\Delta \approx 0.03 R$ the agglomerate is not affected by the entrainment force of the flow, and this zone is agglomerated by inertia. We estimate the value of the final rate of approach of the agglomerate to the wall. We denote by $x=R-y$, the speed of the approaching near-wall zone $\Delta U_{\Delta}$, falling speed on a wall in the normal direction $\Delta U_{\text {wa }}$ the equation of a decelerated motion of agglomerate in the radial direction has an appearance $m \frac{d \Delta U}{d t}=\xi \frac{\pi \delta_{\mathrm{a}}^{2}}{4} \frac{\Delta U^{2}}{2} \rho, m=\frac{\pi \delta_{\mathrm{a}}^{3}}{6} \rho_{\mathrm{a}}, \Delta U=\frac{d x}{d t}, \frac{d \Delta U}{d t}=\frac{d \Delta U^{2}}{2 d x}$. After integration, we will receive the following expression

$$
\frac{\Delta U_{\text {wa }}}{\Delta U_{\Delta}}=\sqrt{\exp \left(-\frac{3}{2} \xi \frac{\rho}{\rho_{\mathrm{a}}} \frac{\Delta}{2 \delta_{\mathrm{a}}}\right)}
$$

We will consider the simplest process of consolidation of agglomerate at his normal contact interaction with a barrier. Problems of the process of deformation and destruction of a rigid-plastic body (striker) when struck against a rigid obstacle are given in [7]. The difference between the processes in the interaction of a dispersed body with an obstacle from the process of interaction of a rigid-plastic body lies in the variability of its density. Experiments show that, at impact rates above a certain value, the agglomerate breaks down to form fragments and adherent fragments; at lower velocities, the agglomerate reduces its length, compacts and adheres to the surface. The agglomerate in the form of a cylinder produces a flat-ended impact on the surface of a motionless absolutely rigid barrier. The impact velocity $\mathrm{V}_{0}$ is directed along the cylinder axis perpendicular to the obstacle plane. The impact process is accompanied by the compression of a dispersed medium with a small change in the cross-sectional area. This can occur in the case of the interaction of particles inside the cylinder, in which each contact is inelastic, and the particles are attracted to each other by autohesive forces. This is typical for particles smaller than $10(\mu \mathrm{m})$. The initial length of the cylinder is L1, the length in the compressed state is L2 (Fig. 2). Upon impact, the dispersed medium from a volume with a length L1-L2 passes into a volume with a length L2. Suppose that before the impact and after impact, the density of the medium in the volumes is distributed uniformly. The condition of preservation of mass of the disperse environment in position of cylinders 1 and 2 gives the following of ratios:

$$
\rho_{1} L_{1}=\rho_{2} L_{2}
$$

The equation of pulses in the interaction of the agglomerate with the wall has the form $\rho_{1} L_{1} \frac{d V}{d t}=\sigma_{\mathrm{s}}, d t=\frac{d l}{V_{0}}$, where $t-$ is a variable time, $l$ - variable length, $V$ - variable speed, 
$\sigma_{\mathrm{s}}-$ contact voltage on the wall. The last equation can be written in the difference form $\rho_{1} L_{1} \frac{d V_{0}^{2}}{\Delta l}=\sigma_{\mathrm{s}}$. Separating the factors and taking into account that $\Delta l=L_{1}-L_{2}$, using equality (14), we obtain the relation between density, velocity, and voltage.

$$
\sigma_{\mathrm{s}}=V_{0}^{2} \frac{\rho_{1}}{l \frac{\rho_{1}}{\rho_{2}}} .
$$

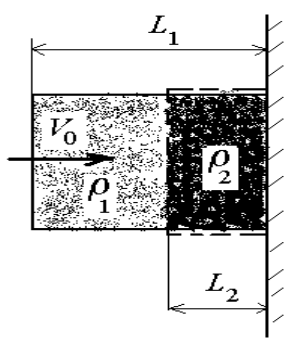

Fig. 2. Scheme of interaction of a cylindrical agglomerate with a surface at right angles.

The density of agglomerate defines its strength parameters, which, in turn, are defined by autohesive internal forces. Therefore, the assumption of a constant density of the dispersed medium in the agglomerate leads to the assumption of a constant stress, which determines the strength during the interaction period. It follows from equation (3) that the contact voltage, which under the above assumptions is also the voltage in the agglomerate at the end of the contact period, is determined by the density and velocity of the agglomerate prior to impact and the density after impact. However, it is clear that the voltage inside the agglomerate should decrease to the free end, so the length-averaged voltage should be less than the voltage at the end. We represent the stress distribution along the length in a volume with the length $\mathrm{L}_{2}$ in the form

$$
\sigma=\sigma_{\mathrm{s}}\left(1-\frac{l}{L_{2}}\right)^{\mathrm{k}}
$$

with $\bar{\sigma}=\frac{1}{L_{2}} \int_{0}^{L_{2}} \sigma_{\mathrm{s}}\left(1-\frac{l}{L_{2}}\right)^{\mathrm{k}} d l=\frac{\sigma_{\mathrm{s}}}{k+1}$, whence $k=\frac{\sigma_{\mathrm{s}}}{\bar{\sigma}}-1=\frac{\rho_{1}}{\rho_{2}} \frac{2}{\left(1-\frac{\rho_{1}}{\rho_{2}}\right)^{2}}-1$.

Table 1.Results of calculating the speed of agglomerates in the radial direction.

\begin{tabular}{|c|c|c|c|c|c|}
\hline$\delta(\mu \mathrm{m})$ & 50 & 100 & 200 & 400 & 800 \\
\hline $\operatorname{Re}_{\delta}$ & 8.2 & 39.2 & 186 & 607 & 1690 \\
\hline$\Delta U_{\Delta}(\mathrm{m} / \mathrm{s})$ & 2.4 & 5.8 & 13.9 & 23 & 32 \\
\hline$\frac{\Delta U_{\text {wa }}}{\Delta U_{\Delta}}$ & 0.31 & 0.48 & 0.69 & 0.83 & 0.91 \\
\hline \multicolumn{6}{|c|}{$\rho_{2} / \rho_{1}=0.7$} \\
\hline$\sigma_{\mathrm{s}}(\mathrm{kPa})$ & 0.553 & 7.75 & 92 & 364 & 847 \\
\hline \multicolumn{6}{|c|}{$\rho_{2} / \rho_{1}=0.4$} \\
\hline$\sigma_{\mathrm{s}}(\mathrm{kPa})$ & 0.276 & 3.87 & 46 & 182 & 423 \\
\hline
\end{tabular}


After the termination of influence of all loadings (the after-effect period) agglomerate in a new state appears in balance. The agglomerate is attracted by the aerodynamic forces from the side of the flow, the gravitational force, and on the other, attracts the wall force due to the adhesion and autohesion of the particles. In the new state, there is no pressure force at the free end, whereas at the contact end there are adhesion forces that depend on the "clamping" force during the interaction, the surface forces acting on the lateral surface are acted upon by autohesive forces. Formula (13) and formulas $(15,16)$ allow the calculation of the contact stress and evaluate the stress distribution of the agglomerate in the period of its interaction with the wall. The calculation is carried out for two values of the ratios of the densities $\rho_{2} / \rho_{1}=0.7$ and $\rho_{2} / \rho_{1}=0.4$ [8], $\rho_{1}=300\left(\mathrm{~kg} / \mathrm{m}^{3}\right)$. In the formula (3) $\Delta U_{\text {wa }} \equiv V_{0}$

Calculation of stresses in the agglomerate itself (table 1) shows fast reduction of tension by the free end.

\section{Conclusion}

Thus, information on density of deposits can be also information on the speed of approach of agglomerate to a surface and about his weight. Estimates show that the stresses in the interactions of the agglomerate with the surface can reach values comparable to atmospheric pressure, which is the reason for the formation of strong deposits, which in turn are determined by the autohesive bonds of the particles forming the agglomerate.

\section{References}

1. A. D. Zimon, Adhesion of dust and powders (Chemistry, Moscow, 1976)

2. V. G. Levich, Physicochemical hydrodynamics (Fizmatgiz, Moscow, 1959)

3. E. Bulba, N. Ivanova, MATEC Web Conf. 141, 01014 (2017)

4. E. Bulba, N. Ivanova, MATEC Web Conf. 110, 01020 (2017)

5. M. V. Vasilevsky, E. G Zykov, Raschet effektivnosti ochistki gaza v inertsial'nykh transportnykh sredstvakh (TPU Publishing House, Tomsk, 2005) [In Russian]

6. A. N. Shtym, Aerodynamics of cyclone-vortex chambers (Far Eastern University, Vladivostok, 1985)

7. A. Y. Sagomonyan, Dynamics of piercing the obstacle (Moscow Univ. Publ., Moscow, 1988)

8. K. V. Nekrasova, A. S. Razva, E. G. Zykov, M. V. Vasilevsky, Energetika: ekologiya, nadezhnost', bezopasnost': materialy dokladov trinadtsatoy Vserossiyskaya nauchnotekhnicheskaya konferentsiya (TPU Publishing, Tomsk, 2007) [In Russian] 